\title{
Dropwise Evaporative Cooling of a Low Thermal Conductivity Solid
}

\author{
M. DI MARZO, Y. LIAO and P. TARTARINI \\ Mechanical Engineering Department \\ University of Maryland \\ College Park, Maryland 20742, USA \\ D. EVANS and H. BAUM \\ Building and Fire Research Laboratory \\ National Institute of Standards and Technology \\ Gaithersburg, Maryland 20899, USA
}

\begin{abstract}
Insight on extinguishment of a solid fuel fire by sprinkler generated droplets is obtained by detailed modelling of a single droplet evaporative cooling on a hot low thermal conductivity solid. The assumption of constant and uniform temperature at the solid-liquid interface, which decouples the solid and the liquid modelling, cannot be applied to this case because strong local cooling of the solid requires the solutions of. both regions (liquid and solid) to be coupled. The large thermal gradients observed at the edge of the droplet preclude the application of finite difference techniques for the integration of the transient conduction governing equation. A mixed technique that uses a control volume method for the liquid and a boundary element formulation for the solid is proposed. Both methods are briefly outlined and the computed predictions are validated with experimental measurements which encompass high resolution thermography of the solid surface subjected to evaporative cooling. Insight on the temperature distribution at the solid-liquid interface is obtained deduced from the model and the deviation from the constant and uniform temperature at the liquid-solid interface is assessed. The radial versus axial conduction in the liquid droplet is also quantified.
\end{abstract}

KEYWORDS: evaporation, cooling, drops

\section{INTRODUCTION}

Local cooling induced by an evaporating droplet deposited on a hot solid surface is investigated. The long term objective of this study is the construction of a model for the prediction and optimization of sprinkler based extinguishment systems performance. In particular, the prediction of evaporative phenomena is considered here, which implies that nucleate boiling at the solid-liquid interface under the droplet is fully suppressed. Note that the evaporative phenomena occur at surface temperatures lower than the one 
obtained by direct exposure to flame radiation. However, evaporation is observed on compartment surfaces exposed to fire (walls, ceiling, furniture, etc.). For the case of high thermal conductivity solids, a simple model $[1,2]$ was based on the assumption that the temperature at the solid-liquid interface is constant and uniform during the evaporation process as suggested by Michiyoshi and Makino [3] and Seki [4].

Fire safety applications are mostly involved with low conductivity materials. For this case the liquid and solid solutions must be coupled since the thermal conditions under the droplet are non-uniform and vary during the evaporation process. The thermal gradients in the proximity of the droplet edge are very large in the initial portion of the transient. This fact precludes the application of finite difference techniques to integrate the transient conduction governing equation. Pronounced numerical instabilities were observed at the edge of the droplet in the solid region. These numerical instabilities could be controlled by reducing the temporal step size. However, the round-off error consequent to the small temporal increments made the solution unacceptable.

A different solution scheme is formulated for the solid region which is based on Boundary Element Methods (BEM). BEM are applied to a number of conduction problems by Carslaw and Jaeger [5]. More recently a review by Pina and Fernandez [6] specifically described BEM applications to transient heat conduction. A very important contribution by Wrobel and Brebbia [7] focuses on the axisymmetric geometry which is the case of this study. Their formulation is different from the one proposed here because it employs a series expansion in time as opposed to the temporal discretization used in this paper. The BEM formally requires that all past information must contribute to the present solution. The advantage of the time discretization scheme presented here, is that only a limited amount of past information must be collected in order to obtain the solution. In the limit it can be shown that the method reduces to an explicit scheme and all the past contributions become negligible.

The complex geometry of the liquid droplet and the future plan to incorporate radiative fluxes into the liquid solution suggest that a Control Volume Method (CVM) be used for the integration of the transient conduction governing equation in the liquid region. A simple nodalization scheme is presented and the governing equation is discretized for each elementary control volume.

\section{MODEL FORMULATION}

The transient conduction equation is applied to the solid region and to the liquid which is considered motionless. In general, the governing equation is written for both the liquid and the solid (with the appropriate thermal diffusivity) as:

$$
\frac{\partial T}{\partial t}=\alpha \nabla^{2} T
$$

The boundary conditions can be summarized as (see Fig 1):

at $z=0 ; r \leq R$

$$
k_{s} \nabla T_{s} \cdot \hat{n}=k_{l} \nabla T_{l} \cdot \hat{n}
$$




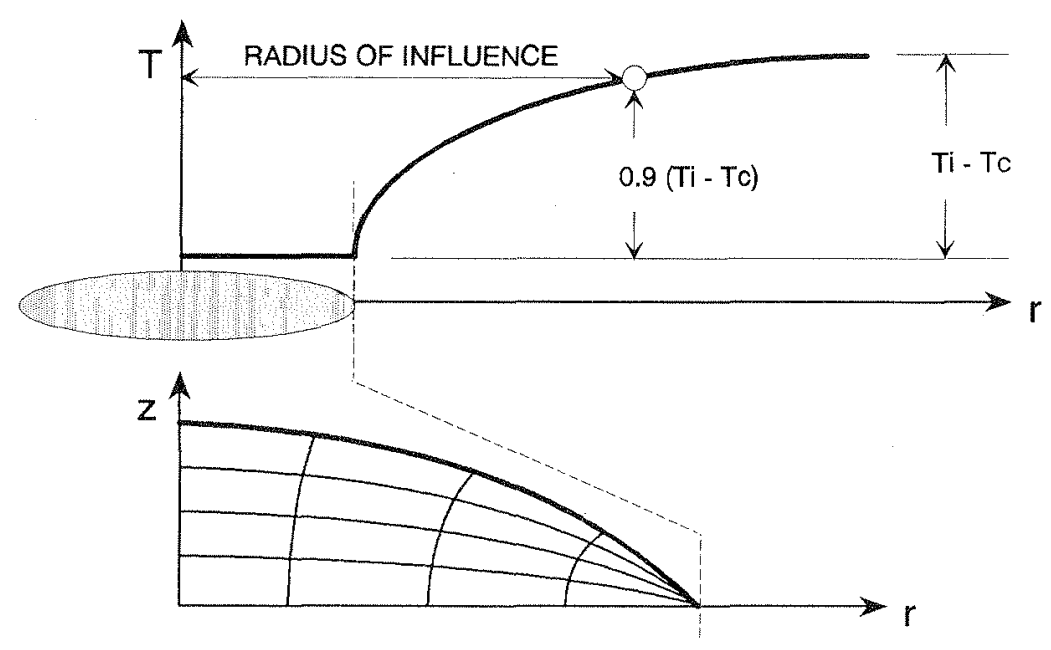

FIGURE 1 - Coordinate system, radius of influence and nodalization

$$
T_{s}=T_{l}
$$

at $z=0 ; r>R$

$$
-k_{s} \nabla T_{s}=h_{a}\left(T_{s}-T_{a}\right)+\sigma \varepsilon\left(T_{s}^{4}-T_{a}^{4}\right)
$$

at $z=f(r)$ liquid-vapor interface

$$
-k_{l} \nabla T_{l} \cdot \hat{n}+h_{a}\left(T_{a}-T_{l}\right)=0.624 h_{a} L e \frac{h_{f g}}{c_{p a}} \frac{x_{i}-x_{a}}{1-x_{i}}
$$

Here $x_{\mathrm{i}}$ and $x_{\mathrm{a}}$ are molar fractions (see the nomenclature for definitions) the radiant heat transfer component and the density variations in the air-vapor mixture at the liquid-vapor interface are neglected. The function $f(r)$ which describes the liquid-vapor interface is a segment of a sphere and is provided in detail in reference [2]. The overall and convective heat transfer coefficients are derived from experimental measures $[1,2]$. The axisymmetric nature of the problem requires that the radial gradient of $T$ is zero on the vertical axis through the origin of the coordinate system. The initial condition will be either a linear one-dimensional temperature distribution in the solid and uniform temperature in the liquid or uniform temperatures in both liquid and solid. Note that the initial contact temperature at the liquid-solid interface is set to the contact temperature $T_{c}$ defined by Seki [4]. This contact temperature depends on the water and solid properties and on their respective initial temperatures; therefore, it is a constant reference value. Initially, the water is considered at the ambient temperature $\left(T_{a}\right)$. 
The use of a BEM is desirable because the solution at a given point is obtained by superimposing all the heat flux contributions from the neighboring points; hence the localized, drastic thermal changes of this cooling process are smoothed out. When a finite difference technique is used the sharp gradients are locally amplified thus causing the observed instabilities in the solution unless an extremely fine nodalization is used. Furthermore, the computation is limited to the surface points and allows a more precise definition of the noding in the region of concern (e.g., at the droplet outer edge).

To obtain the desired formulation, it is necessary to introduce an adjoint equation to Eq. (1) in terms of the Green's function $G\left(\vec{v}, \vec{v}_{0}, t, t_{0}\right)$; that is

$$
\frac{\partial G}{\partial t}=-\alpha \nabla^{2} G
$$

where the vector position $\vec{v}$ is in terms of any specified coordinate system. Note that $G$ depends on the point $\vec{v}$ which is the point of interest and on the point $\vec{v}_{0}$ which is the generic point identified by the integration coordinates. Similarly, a backward time scale $t_{0}$ must be introduced. The origin of this time scale is at the present time $t$ and the time $t_{\mathrm{o}}$ increases in the negative direction of actual time. In what follows, $t_{0}$ is identified as the recollection time. The need for this dual time scale is required by the definition of the adjoint equation which carries a negative sign on the right hand side. By multiplying Eq. (1) by $G$ and Eq. (6) by $T$, respectively, and integrating over the domain with Gauss' theorem, one obtains:

$$
\iint_{t} \frac{\partial(T G)}{\partial t} d v_{0} d t_{0}=\alpha \int_{t} \oint_{s}(T \nabla G-G \nabla T) \cdot \hat{n} d s_{0} d t_{0}
$$

At this point the left-hand-side is expanded into two volume integrals at the present time and at initial time, respectively. One of the properties of the function $G$ is that as the recollection time goes to zero $\left(t_{0} \rightarrow 0\right), G$ becomes the Dirac function. Therefore, the volume integral at the present time reduces to $T$. Further, one can introduce a new variable $u$ in lieu of the solid temperature $T$ such that it is zero in all the volume at the initial time. For instance, for the solid region subjected to constant heat flux in the axial direction, one can define $u$ as:

$$
u=T-T_{i}+\frac{q z}{k_{l}}
$$

With these two modifications and by selecting a function $G$ such that $D G$ is zero at $z=0$, the final result is achieved in the form:

$$
u=\alpha \iint_{t} G \nabla u \cdot \hat{n} d s_{0} d t_{0}
$$

This integral is performed over a closed surface. However, the surface bounding the solid region below the plane $z=0$ is far ahead of the advancing thermal wave. Therefore, the contribution of the points on the surface bounding the domain in the far 
field is negligible. With the implementation of the cylindrical coordinate system, the equation is further simplified since the angular integration can be expressed in terms of a Bessel function. The final form results in a double integral in time and along the radius $r$ which can be cast in the following form:

$$
u(r, t)=\frac{1}{\sqrt{4 \pi \alpha}} \int_{0}^{t} \int_{0}^{\infty} \nabla u\left(r_{0}, t-t_{0}\right) r_{0} t_{0}^{-\frac{3}{2}} L_{0}\left(\frac{2 r r_{0}}{4 \alpha t_{0}}\right) e^{-\frac{\left(r^{2}-r_{0}^{2}\right)}{4 \alpha t_{0}}} d r_{0} d t_{0}
$$

In order to simplify the task of handling these complex surface integrals, a decomposition in two portions is proposed: the value of the forcing function or of the unknown function $(\nabla u)$ is assumed to be constant in the small intervals $d r_{\circ}$ and $d t_{\circ}$. This introduces formal errors of the order $\left(d r_{\mathrm{o}} / R\right)^{2}$, and $\left(d t_{\mathrm{o}} / t\right)^{2}$ into the analysis. The various surface integrals can be recast in the form:

$$
u=\sum_{i=1}^{n} W_{i} \cdot \vec{f}_{i}+W_{0} \cdot \vec{f}_{0}
$$

where $W$ is a weight matrix and $\vec{f}$ is the vector of the forcing and unknown functions. The summation term is known since it involves previously calculated parameters. The second term on the right-hand-side of Eq. (11) contains unknown functions or the specified boundary conditions.

The effect of the forcing function at the point of concern and the singular behavior of points at the origin of the spacial and temporal coordinates must be considered. The first particular case of interest is when the point of concern coincides with the source point. This corresponds to the maximum influence of a source point because the distance between the two points is minimal. In terms of weight this yields:

$$
W\left(r \equiv r_{0}, t_{0}\right)=r t_{0}^{-1} L_{0}\left(\frac{2 r^{2}}{4 \alpha t_{0}}\right) \operatorname{erf}\left(\frac{\Delta r_{0}}{\sqrt{16 \alpha t_{0}}}\right) \Delta t_{0}
$$

Note that the term $\Delta t_{\mathrm{o}}$ is consistent with the formulation of Eq. (12) where the forcing function $f$ is multiplied by the weight $W$. By comparing Eq. (12) with the general formulations for $u$ [Eq. (10)] it is clear that a temporal and spacial integration must be performed on the weighing function. The integration in this case is performed analytically in space and numerically in time. The units of the weight $W$ are those of length as expected.

The singularities at the origin of the spacial and temporal coordinates are addressed by staggering the discretization of the domain. The region neighboring the axis of symmetry is handled by locating the node nearest the symmetry axis at $\Delta r_{d} / 2$. Similarly, the time discretization is not carried to the recollection time origin (i.e. the present time or $t_{\mathrm{o}}=0$ ).

As far as the liquid region is concerned a Control Volume Method (CVM) is used to discretize the governing equation and its boundary conditions. The droplet 

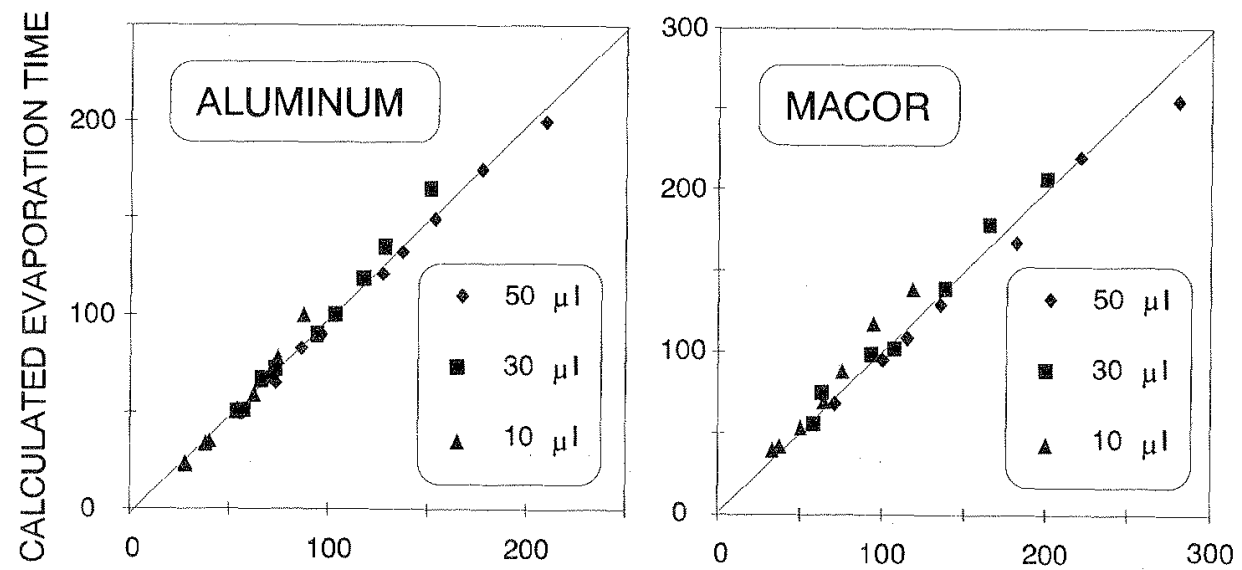

EXPERIMENTAL EVAPORATION TIME

FIGURE 2 - Evaporation time: computations versus measurements

shape is described as a segment of a sphere [1]. The nodalization scheme consists of two sets of curves: a) arcs of circumference passing through the outer edge of the droplet (at $z=0$ ) and centered on the $z$ axis and $b$ ) arcs of circumference centered on $z=0$ with radii inversely proportional to the distance between the origin and the intercept of each arc with the $r$ axis (see Fig. 1).

The governing equation integrated over each elementary control volume reduces to a transient energy balance where each incoming flux is evaluated with respect to its component normal to the surface bounding the elementary volume. The nodalization of the outer edge of the droplet is carried out by locating the nodes at $\Delta r / 2$ from the droplet edge. Particular care is taken in the integration of the heat and mass transfer fluxes at the droplet edge to account for the sharp changes in temperature in that region.

\section{MODEL VALIDATION}

The predictions of the coupled solid-liquid model are compared with experimental data for aluminum and Macor. Macor is a glass-like material which is able to withstand strong local thermal stress. The properties of these two materials are listed in TABLE 1.

TABLE 1 - Solid Properties

Specific Heat $\left(\mathrm{J} / \mathrm{Kg}^{\circ} \mathrm{C}\right)$

Thermal Conductivity $\left(\mathrm{W} / \mathrm{m}^{\circ} \mathrm{C}\right)$

Thermal Diffusivity $\left(\mathrm{m}^{2} / \mathrm{s}\right)$

Total Emittance

Aluminum [8]

962

180

$4.55 \times 10^{-5}$

0.08
Macor [9]

835

1.29

$6.19 \times 10^{-7}$

0.94 


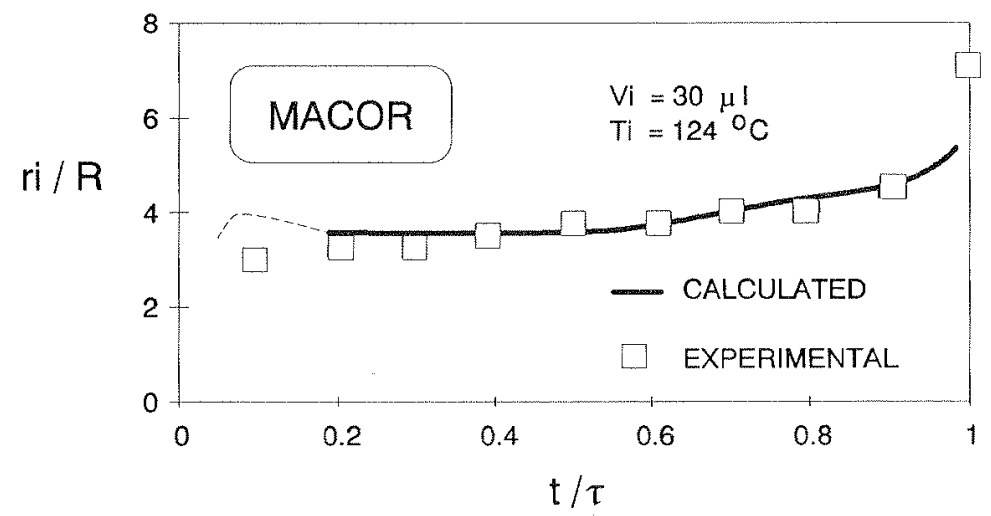

FIGURE 3 - Radius of influence versus time: computations versus measurements

Consider the difference of two orders of magnitude in the thermal properties and note that the model is able to predict the evaporation time for both cases with reasonable agreement with the experimental data as shown in Fig.2.

Some discrepancies are observed for high evaporation time, that is for the low temperature cases. Such discrepancies are within the fifteen per cent band. This error band is to be expected in light of the uncertainties involved in the determination of the mass transfer coefficient and the materials thermal properties. The droplet size range is one order of magnitude larger than the average sprinkler droplet size. However, the smaller sprinkler generated droplets might not be able to reach the solid surface due to up-drafts and/or evaporation, hence the droplet size distribution is skewed towards the higher sizes.

To further validate the model, the radius of influence during the evaporation of a water droplet on Macor is calculated and compared with the radius of influence behavior deduced from the infrared thermography of the solid surface. Note that this comparison involves temperature profiles on the solid surface at various times during the transient. Figure 3 demonstrates the model capabilities. In the initial portion of the transient, the sudden contact of solid and liquid with large temperature differences causes a substantial dip in the solid temperature in the experiments and an overshoot of the radius of influence in the model (as can be seen in the dashed line portion of the curve). Further refining of the model is needed to reconcile these discrepancies. However, the contribution of the initial portion of the transient does not affect significantly the overall results.

\section{RESULTS AND CONCLUSIONS}

The detailed model presented here provides an understanding of the limitations associated with the simplified model for high thermal conductivity solid described in earlier works [1,2]. This simplified model is based on the assumption of uniform and constant temperature at the liquid-solid interface and on the dominance of the axial component of the flux over its radial component in the liquid droplet heat transfer.

Figure 4 shows typical temperature profiles on the solid surface. Note that for aluminum all the variations in temperature span less than one degree centigrade, while 


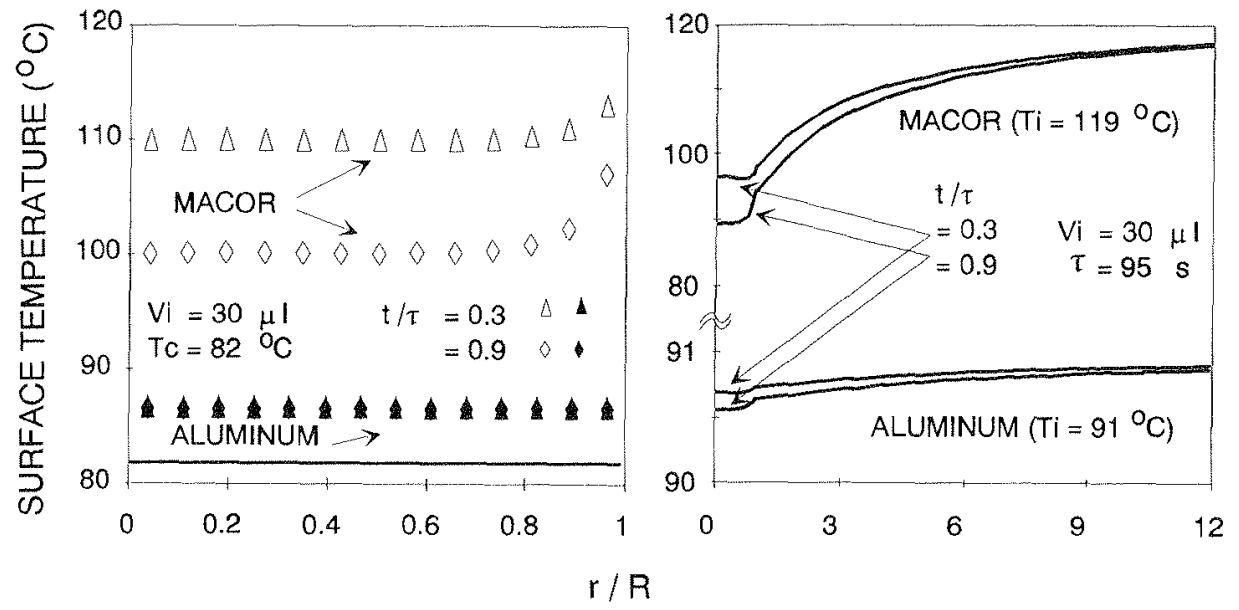

FIGURE 4 - Calculated solid surface temperature profiles on Macor and Aluminum

for Macor the temperatures vary almost $30^{\circ} \mathrm{C}$. This fact is further elucidated in the plot (to the left) at the solid-liquid interface which is shown for various times and locations under the droplet. The results presented in the plot to the left are for a $30 \mu \mathrm{l}$ droplet which, deposited both on Macor $\left(\right.$ at $\left.T_{\mathrm{i}}=140^{\circ} \mathrm{C} ; \tau=66 \mathrm{~s}\right)$ and aluminum (at $T_{\mathrm{i}}=87^{\circ} \mathrm{C} ; \tau=105 \mathrm{~s}$ ), generates the same calculated contact temperature of $82^{\circ} \mathrm{C}$. It is clear that the hypothesis of uniform and constant temperature at the solid-liquid interface is excellent for aluminum and unacceptable for Macor.

Further, in the simplified model for high conductivity solids, the interfacial temperature was assumed equal to the contact temperature. This assumption is rather fortuitous in that the error made is within ten per cent, notwithstanding the lack of fundamental basis for the correlation between these two temperatures.

Figure 5 shows the relative magnitude of the axial and radial component of the heat flux for various droplet sizes, solid materials, initial solid surface temperatures, locations within the droplet and elapsed evaporation time. These results show that the assumption of dominant axial conduction is reasonable since the axial heat flux is more than 90 per cent of the total heat flux in most cases. The points to the right side of the figure are taken at the droplet edge for Macor and show axial heat fluxes of slightly less than 90 per cent of the total heat flux. This result is to be expected considering the temperature distribution at the edge of the liquid-solid interface as depicted in Fig. 4.

In conclusion, a powerful and complex model for the prediction of the thermal transient behavior of water droplets deposited on hot solids is demonstrated. Some of the computed results provide insight on the limits of the simplified model presented earlier [1,2]. Better understanding of the processes involved in dropwise evaporative cooling is achieved. Further work to resolve the details of the initial portion of the thermal transient is required although its contribution to the total evaporation process is small [2]. 


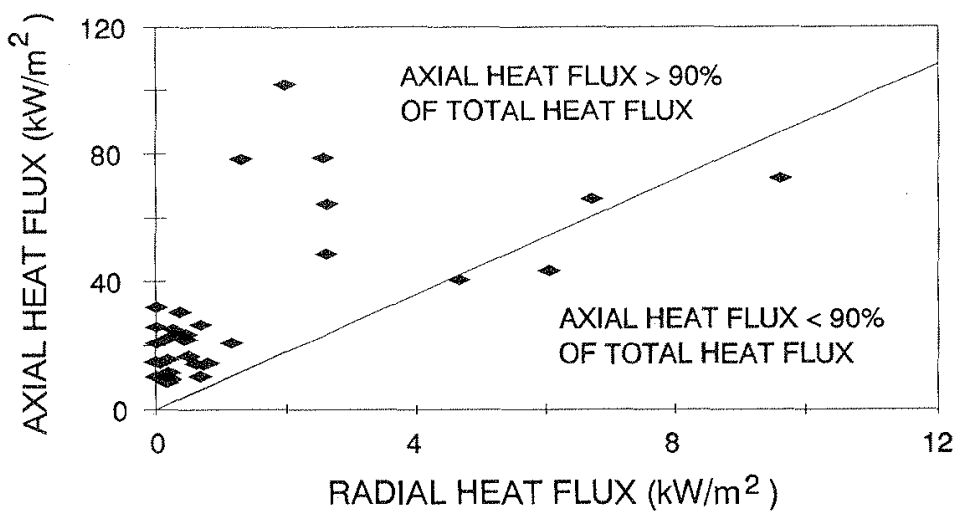

FIGURE 5 - Calculated axial and radial heat flux components

\section{ACKNOWLEDGEMENTS}

This research is supported by a grant of the National Institute for Standards and Technology with contributions by the Computer Science Center of the University of Maryland. The authors wish to acknowledge the contribution of Mr. F. Kavoosi for the BEM formulation and Mr. M. Klassen for the infrared thermography.

\section{NOMENCLATURE}

$\begin{array}{ll}c_{p} & \text { specific heat } \\ \mathrm{D} & \text { steam water mass diffusivity } \\ \mathrm{f} & \text { forcing and unknown vector } \\ \mathrm{G} & \text { Green's function } \\ \mathrm{h}_{\mathrm{a}} & \text { convective heat transfer coefficient }\left(\propto 12 \mathrm{~W} / \mathrm{m}^{2}{ }^{\circ} \mathrm{C} \text {, see }[8]\right) \\ \mathrm{h}_{\mathrm{fg}} & \text { latent heat of vaporization } \\ \mathrm{k} & \text { thermal conductivity } \\ \mathrm{L}_{\mathrm{o}}(\mathrm{z}) & \text { Bessel function: } \mathrm{e}^{-2} \mathrm{I}(\mathrm{z}) \\ \mathrm{L} & \text { Lewis number: }(\mathrm{D} / \alpha)^{2 / 3} \\ \mathrm{n} & \text { unit vector normal to the surface } \\ \mathrm{q} & \text { heat flux } \\ \mathrm{r} & \text { radial coordinate } \\ \mathrm{r}_{\mathrm{j}} & \text { radius of influence (see Fig. 1) } \\ \mathrm{R} & \text { radius of the solid wetted region } \\ \mathrm{s} & \text { surface } \\ \mathrm{t} & \text { time } \\ \mathrm{t}_{\mathrm{o}} & \text { recollection time } \\ \mathrm{T} & \text { temperature } \\ \mathrm{T}_{\mathrm{a}} & \text { far tield air temperature } \\ \mathrm{T}_{\mathrm{c}} & \text { contact temperature defined by Seki }[4] \\ \mathrm{T}_{\mathrm{i}} & \text { initial solid surface temperature } \\ \mathrm{u} & \text { transformed temperature } \\ \mathrm{V} & \text { volume } \\ \mathrm{V} & \text { position vector } \\ \mathrm{V}_{\mathrm{i}} & \text { initial droplet volume } \\ \mathrm{W} & \text { weight matrix }\end{array}$




$\begin{array}{ll}\mathrm{X}_{\mathrm{a}} & \begin{array}{l}\text { far field steam in air molar fraction } \\ \text { interfacial steam in air molar fraction } \\ \text { axial coordinate }\end{array} \\ \mathrm{z} & \text { thermal diffusivity } \\ \alpha & \text { spatial step } \\ \Delta \mathrm{r}_{\mathrm{o}} & \text { time step } \\ \Delta \mathrm{t}_{\mathrm{o}} & \text { solid surface total emittance } \\ \epsilon & \text { Stefan-Boltzmann constant } \\ \sigma & \text { total evaporation time }\end{array}$

Subscripts:

$\begin{array}{ll}1 & \text { liquid } \\ \circ & \text { generic point index } \\ \mathrm{r} & \text { in the radial direction } \\ \mathrm{s} & \text { solid } \\ \mathrm{z} & \text { in the axial direction }\end{array}$

\section{REFERENCES}

1. diMarzo, M. and Evans, D.D., "Dropwise Evaporative Cooling of High Thermal Conductivity Materials", Heat and Technology, 5: 1-2, 126-136, 1987.

2. diMarzo, M. and Evans, D.D., "Evaporation of a Water Droplet Deposited on a Hot High Thermal Conductivity Solid Surface", ASME Journal of Heat Transfer, 111: 210-213, 1988.

3. Michiyoshi, I. and Makino, K., "Heat Transfer Characteristics of Evaporation of a Liquid Droplet on Heated Surfaces", International Journal of Heat and Mass Transfer, 21: 605-613, 1978.

4. Seki, M., Kawamura, H. and Sanokawa, K., "Transient Temperature Profile of a Hot Wall Due to an Impinging Liquid Droplet", ASME Journal of Heat Transfer, 100: 167-169, 1978.

5. Carslaw, H.S. and Jaeger, J.C., Conduction of Heat in Solids, Oxford University Press, 1959.

6. Pina, H.L.G. and Fernandes, J.L.M., "Applications in Transient Heat Conduction", Topics in Boundary Element Research, ed. C.A. Brebbia, 1: 2, pp. 41-58, Springer-Verlag, 1984.

7. Wrobel, L.C. and Brebbia, C.A., "A formulation of the Boundary Element Method for Axisymmetric Transient Heat Conduction", International Journal of Heat and Mass Transfer, 24: 843-850, 1981.

8. Chapman, A.J., Heat Transfer Macmillan Publishing Co., 1984.

9. Corning, Macor Machinable Glass Ceramic Manufacturer Literature 